\title{
Future Prospects of Point Fluorescence Spectroscopy, Fluorescence Imaging and Fluorescence Endoscopy in Photodynamic Therapy (PDT) for Cancer Cells
}

\section{A Heidari*}

California South University, 14731 Comet St. Irvine, CA 92604, USA

Photodynamic Therapy (PDT) has been developing rapidly within various medical specialties since the 1980s. It matured as a feasible medical technology in the 1980s thought the world. Photodynamic Therapy (PDT) includes two steps of process. The first step is administration of a photosensitizer (PS) and the second step is activation by light lamination at an appropriate wave length to reactive the chemicals, causing them to emit free radicals and destroy the targeted abnormal cells. Also, Photodynamic Therapy (PDT) has been used for palliation and curative treatment. It used in treatment of esophageal cancer, stomach cancer, cholangiocarcinoma and barrett. Although Photodynamic Therapy (PDT) is a safe and efficient method, it still has not become a first line procedure. It should not be used in acute porphyria, poor kidney and liver function, pregnancy and breast feeding. Iatrogenic porphyria that may cause cutaneous erythema, edema or blistering is one of the major complications [1-16].

The future of Photodynamic Therapy (PDT) will build on our scientific and clinical success, expand to new horizons. Although Photodynamic Therapy (PDT) developed as a tumor therapy method but it is now becoming widely used in medicine and biology. Researchers in Photodynamic Therapy (PDT) in particular are relentlessly exponential. Some of the main aspects of research including new and better drugs that able to treat tumors at deeper under the skin or in body tissues, more selective for cancer cells, collect in cancer cells and also remove from the body more quickly. Other light sources at different types of lasers and light sources for small doses of radiation which could lead to fewer side effects.

Combining treatment with other therapeutic modalities may give considerable improvement of treatment efficiency over monotherapy alone. Elucidation of cellular mechanisms, signaling and death pathways; together with findings concerning processes that occur in tumors involving oxidative stress, inflammation and dead cell disposal are active areas of research that may lead to insights into how the host response to Photodynamic Therapy (PDT) activities the immune system.

Although the use of covalent photosensitizer (PS) conjugates to increase tumor and other tissues targeting is very active area of preclinical research, the applications of Photodynamic Therapy (PDT) to a number of novel preclinical and clinical applications such as bone, blood vessels and for infections will increase the interest of the bio analytical and biomedical communities in Photodynamic Therapy (PDT) as a whole.

A photosensitizer (PS) is defined as a chemical compound, which upon absorption of light induces a chemical and physical alteration of another chemical compound. A good photosensitizer (PS) should absorb photons efficiently, have a high quantum yield of triplet formation and the triplet state should be long lived in order to have time to react with neighboring target molecules. Most compounds that form triplet states that are able to produce radicals and reactive Oxygen species have a tricyclic, heterocyclic or porphyrin - like ring structures with conjugated double bands. Small changes in the molecular structure may influence the photosensitising property of a compound. Therefore, Photodynamic Therapy (PDT) can be improved greatly by optimizing photosensitizer (PS) chemical properties via structural modifications. The molecular characteristics of the photosensitizer (PS) such as charge, lipophilicity and asymmetry govern the localization and uptake of the compounds by various cells types and also determine the pharmacokinetics, biodistribution and localization of the photosensitizer (PS) at the target site.

Recently, development of new photosensitizers (PS) with more efficacies has been considered. Among various photosensitizers (PS), tetrapyrrole containing compounds whose macrocyclic moiety plays a key role in generating cytotoxic reactive Oxygen species upon light illumination are specially suitable for chemical modifications of the coordination sphere and at the periphery of the macrocycle. It has been reported that introduction of metal cations into the coordination sphere of tetrapyrrolic compounds conferred an increased photosensitizing potency. Furthermore, phenol and lipophilic groups as the peripheral substituents in chlorins have been reported to be therapeutically relevant.

On the other hand, the presence of fungal infection in oral cavity has been increased due to the increase of the using antineoplastic and immunosuppressive drugs, using antibiotics and wearing prosthetic devices. Photodynamic Therapy (PDT) is a new modality for treatment of fungal infection. After application of photosensitizers (PS) to the fungal site, laser light at a specific wavelength is activated which destroys the target cell through the production of singlet Oxygen. Using Photodynamic Therapy (PDT) for treatment of oral infection has more advantages than traditional antifungal drug therapy. Photodynamic Therapy (PDT) has the great potential for treating oral fungal infection and can be considered as an appropriate alternative treatment.

Photodynamic Diagnosis (PDD) or Fluorescence Diagnosis (FD) is designed on detection of premalignant lesions and cancer tumors through recording and evaluation of fluorescence emission profile. Photodynamic Diagnosis (PDD) is a modern method and a minimally invasive approach based on one of these processes: Autofluorescence of tissues, fluorescence of a chemiluminescence probe or fluorescence after local or systemic administration of a photosensitizer (PS),

*Corresponding author: A Heidari, Faculty of Chemistry, California South University (CSU), 14731 Comet St. Irvine, CA 92604, USA, Tel: +1-775-410-4974 E-mail: Scholar.Researcher.Scientist@gmail.com

Received March 29, 2016; Accepted March 30, 2016; Published April 04, 2016

Citation: Heidari A (2016) Future Prospects of Point Fluorescence Spectroscopy Fluorescence Imaging and Fluorescence Endoscopy in Photodynamic Therapy (PDT) for Cancer Cells. J Bioanal Biomed 8: e135. doi:10.4172/1948593X.1000e135

Copyright: (c) 2016 Heidari A. This is an open-access article distributed under the terms of the Creative Commons Attribution License, which permits unrestricted use, distribution, and reproduction in any medium, provided the original author and source are credited. 
Citation: Heidari A (2016) Future Prospects of Point Fluorescence Spectroscopy, Fluorescence Imaging and Fluorescence Endoscopy in Photodynamic Therapy (PDT) for Cancer Cells. J Bioanal Biomed 8: e135. doi:10.4172/1948-593X.1000e135

selectively accumulating in pathological foci. Necessary conditions for successful Photodynamic Diagnosis (PDD) are selectivity and good uptake of photosensitizer (PS) by the pathologic cells and providing possibility of real -time data processing, additionally high precision and sensitivity in optical parameters measurements.

Most important specifications of an appropriate photosensitizer (PS) are proper chemical structure and localization into the tumor, strong emission peak at transparent optical window, high quantum yield and rapid clearance. 5 -Aminolevulinic acid (5 - ALA), Mono - L - aspartyl chlorin e6 (Npe6), Meta - tetra (hydroxyphenyl) chlorin (mTHPC) and Hypericin are the photosensitizing agents used in Photodynamic Diagnosis (PDD).

Photodynamic Diagnosis (PDD) can be utilized in photosensitizer (PS) localization in tumor to determine optimum time for lesion illuminating after dye administration in photodynamic therapy, photobleaching detection, detecting singlet Oxygen using a chemiluminescence probe, Fluorescence - Guided Resection (FGR) and finally in vivo studies for diagnosis of early malignancies and tumors of skin, colon, bronchi, breast, bladder, and female genital tract. Different approaches are used in recording of data such as point fluorescence spectroscopy, fluorescence imaging and fluorescence endoscopy.

There are various challenges that should be considered in the future prospects such as developing new targeted photosensitizer (PS) in order to provide more selectivity in tumor cells and deeper treatment and diagnosis and also, designing imaging and data recording systems for different applications associated with real - time data processing software.

\section{References}

1. Ahn PH, Quon H, O'Malley BW, Weinstein G, Chalian A, et al. (2016) Toxicities and early outcomes in a phase 1 trial of photodynamic therapy for premalignant and early stage head and neck tumors. Oral Oncology 55: 37-42.

2. Neumann LM, Beseoglu K, Slotty PJ, Senger B, Kamp MA, et al. (2016) Efficacy of 5 - aminolevulinic acid based photodynamic therapy in pituitary adenomas - experimental study on rat and human cell cultures. Photodiagnosis and Photodynamic Therapy 14: 77-83.

3. Gomes Filho JE, Araujo GS, Sipert CR, Santos LMDS, Queiroz IODA, et al. (2016) Evaluation of photodynamic therapy on fibroblast viability and cytokine production. Photodiagnosis and Photodynamic Therapy 13: 97-100.

4. Azaïs H, Schmitt C, Tardivel M, Kerdraon O, Stallivieri A, et al. (2016) Assessment of the specificity of a new folate - targeted photosensitizer for peritoneal metastasis of epithelial ovarian cancer to enable intraperitoneal photodynamic therapy. A preclinical study, Photodiagnosis and Photodynamic Therapy 13: 130-138.

5. Ribeiro JBP, Miranda Vilela AL, Graziani D, Gomes MRDA, Amorim AAS, et al. (2016) Evaluation of the efficacy of systemic miltefosine associated with photodynamic therapy with liposomal chloroaluminium phthalocyanine in the treatment of cutaneous leishmaniasis caused by Leishmania (L.) amazonensis in C57BL / 6 mice. Photodiagnosis and Photodynamic Therapy 13: 282-290.

6. Soares JA, Soares SMCS, César CAS, Carvalho MARD, Brito Júnior M, et al. (2016) Monitoring the effectiveness of photodynamic therapy with periodic renewal of the photosensitizer on intracanal Enterococcus faecalis biofilms. Photodiagnosis and Photodynamic Therapy 13: 123-127.

7. Feuser PE, Gaspar PC, Jacques AV, Tedesco AC, Silva MCDS, et al (2016) Synthesis of $\mathrm{ZnPc}$ loaded poly(methyl methacrylate) nanoparticles via miniemulsion polymerization for photodynamic therapy in leukemic cells. Materials Science and Engineering: C 60: 458-466.

8. Rossetti FC, Depieri LV, Praça FG, Ciampo JOD, Fantini MCA, et al. (2016) Optimization of protoporphyrin IX skin delivery for topical photodynamic therapy: Nanodispersions of liquid- crystalline phase as nanocarriers. European Journa of Pharmaceutical Sciences 83: 99-108.

9. Dhillon SS, Demmy TL, Yendamuri S, Loewen G, Nwogu C, et al. (2016)A Phase I Study of Light Dose for Photodynamic Therapy Using 2-[1- Hexyloxyethyl]-2 Devinyl Pyropheophorbide - a for the Treatment of Non - Small Cell Carcinoma In Situ or Non - Small Cell Microinvasive Bronchogenic Carcinoma: A Dose Ranging Study. Journal of Thoracic Oncology 11: 234-241.

10. Kamada Y, Murayama Y, Arita T, Kosuga T, Konishi H, et al. (2016) Intraoperative 5 - aminolevulinic acid-mediated photodynamic diagnosis of gallbladder cancer: A case report. Photodiagnosis and Photodynamic Therapy 14: 74-76.

11. Souza TDD, Ziembowicz FI, Müller DF, Lauermann SC, Kloster CL, et al. (2016) Evaluation of photodynamic activity, photostability and in vitro drug release of zinc phthalocyanine-loaded nanocapsules. European Journal of Pharmaceutical Sciences 83: 88-98.

12. Sobotta L, Wierzchowski M, Mierzwicki M, Gdaniec Z, Mielcarek J, et al. (2016) Photochemical studies and nanomolar photodynamic activities of phthalocyanines functionalized with 1,4,7-trioxanonyl moieties at their nonperipheral positions. Journal of Inorganic Biochemistry 155: 76-81.

13. Ogawara KI, Shiraishi T, Araki T, Watanabe TI, Ono T, et al. (2016) Efficient anti-tumor effect of photodynamic treatment with polymeric nanoparticles composed of polyethylene glycol and polylactic acid block copolymer encapsulating hydrophobic porphyrin derivative. European Journal of Pharmaceutical Sciences 82: 154-160.

14. Inoue K, Matsuyama H, Fujimoto K, Hirao Y, Watanabe H, et al. (2016) The clinical trial on the safety and effectiveness of the photodynamic diagnosis of non-muscle-invasive bladder cancer using fluorescent light-guided cystoscopy after oral administration of 5-aminolevulinic acid (5-ALA). Photodiagnosis and Photodynamic Therapy 13: 91-96.

15. Damante CA, Ducati P, Ferreira R, Salmeron S, Zangrando MSR, et al. (2016) In vitro evaluation of adhesion / proliferation of human gingival fibroblasts on demineralized root surfaces by toluidine blue $\mathrm{O}$ in antimicrobial photodynamic therapy. Photodiagnosis and Photodynamic Therapy 13: 303-307.

16. Sellera FP, Gargano RG, Libera AMM, Benesi FJ, Azedo MR, et al. (2016) Antimicrobial photodynamic therapy for caseous lymphadenitis abscesses in sheep: Report of ten cases. Photodiagnosis and Photodynamic Therapy 13 120-122. 